\title{
Utilizing DNase I and graphene oxide modified magnetic nanoparticles for sensing PD-L1 in human plasma
}

\author{
Xudong $\mathrm{He}$ \\ Hubei University of Medicine Affiliated Dongfeng Hospital, Shiyan, China \\ GuangYi Yang, E. Yang and Moli Zhang \\ Shenzhen Baoan Authentic TCM Therapy Hospital, Guangdong Shenzhen, China \\ Dan Luo, Fingïan Liu and Chongnan Zhao \\ Hubei University of Medicine Affiliated Dongfeng Hospital, Shiyan, China \\ Qinhua Chen \\ Shenzhen Baoan Authentic TCM Therapy Hospital, Guangdong Shenzhen, China, and \\ Fengying Ran \\ Hubei University of Medicine Affiliated Dongfeng Hospital, Shiyan, China
}

\begin{abstract}
Purpose - Based on DNase I and reduced graphene oxide ( $\mathrm{rGO}$ )-magnetic silicon microspheres (MNPS), a highly sensitive and selective fluorescent probe for the detection of PD-L1 was developed.

Design/methodology/approach - Here ${ }^{\circ} \mathrm{C}$ we present a feasibility of biosensor to detection of PD-L1 in lung tumors plasma. In the absence of PD$\mathrm{L} 1{ }^{\circ} \mathrm{C}$ the PD-L1 aptamer is absorbed on the surface of graphene oxide modified magnetic nanoparticles ${ }^{\circ} 8 \mathrm{rGO}-\mathrm{MNPS}^{\circ} 9$ and leading to effective fluorescence quenching. Upon adding PD-L1 ${ }^{\circ} \mathrm{C}$ the aptamer sequences could be specifically recognized by PD-L1 and the aptamer/PD-L1 complex is formed ${ }^{\circ} \mathrm{C}$ resulting in the recovery of quenched fluorescence.

Findings - This sensor can detect PD-L1 with a linear range from $100 \mathrm{pg} \mathrm{mL}^{-1}$ to $100 \mathrm{ng} \mathrm{mL}^{-1}$, and a detection limit of $10 \mathrm{pg} \bullet \mathrm{m}^{-1}$ was achieved. Originality/value - This method provides an easy and sensitive method for the detection of PD-L1 and will be beneficial to the early diagnosis and prognosis of tumors.
\end{abstract}

Keywords Biosensors, Sensor networks, Programmed death-ligand 1, Cancer diagnosis, Fluorescence Biosensor

Paper type Research paper

\section{Introduction}

Cancer has a major impact on society across the world; it is the leading cause of death in the worldwide. In 2012, there were 14.1 million new cases of cancer and 8.2 million cancer-related deaths in the worldwide; the number of new cases of cancer per year is expected to rise to 23.6 million by 2030 (www.cancer. gov/about-cancer/understanding/statistics). At present, the main early detection techniques of cancer are low-dose computed tomography, mammography and magnetic resonance imaging, colonoscopy and tissue biopsy, etc. However, its often high cost, low sensitivity, high false positive rate and have invasive procedure (Oeffinger et al., 2015; Patz et al., 2014; Chudgar et al., 2015; Aberle et al., 2013). Therefore, it is urgent to develop a rapid and accurate method for detecting cancer biomarkers.

The current issue and full text archive of this journal is available on Emerald Insight at: https://www.emerald.com/insight/0260-2288.htm

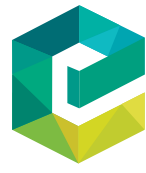

Sensor Review

41/3 (2021) 229-234

Emerald Publishing Limited [ISSN 0260-2288]

[DOI 10.1108/SR-04-2020-0087]
(C) Xudong He, GuangYi Yang, E. Yang, Moli Zhang, Dan Luo, Jingjian Liu, Chongnan Zhao, Qinhua Chen and Fengying Ran. Published by Emerald Publishing Limited. This article is published under the Creative Commons Attribution (CC BY 4.0) licence. Anyone may reproduce, distribute, translate and create derivative works of this article (for both commercial and non-commercial purposes), subject to full attribution to the original publication and authors. The full terms of this licence may be seen at http://creativecommons.org/licences/by/4.0/legalcode

These works were supported by the National Natural Science Foundation of China (81872509), the Hubei Provincial Technology Innovation Project (2017ACA176), the Shennongjia National Park, Hubei Provincial Key Laboratory for Conservation Biology of Snub-nosed Monkeys, open Project Fund of Shennongiia Golden Monkey Conservation Biology Hubei Provincial Key Laboratory (201801), the Free Exploration Project of Hubei University of Medicine (FDFR201804), the Open Project of Hubei Key Laboratory of Wudang Local Chinese Medicine Research (Hubei University of Medicine) (Grant No. WDCM2018004), the Research Project of Traditional Chinese Medicine of the Foundation of Health Commission of Hubei Province (ZY2019M034), the Key Discipline Project of Hubei University of Medicine and Hubei Province health, family planning scientific research project (WJ2019M054), the Science and Technology Key Program of Shiyan (19Y93).

Received 20 April 2020

Revised 6 August 2020

Accepted 30 September 2020 
PD-L1 is an important immunosuppressive molecule (Lieping and Flies, 2013; Okazaki et al.,2013); it has important significance in antitumor, anti-infection, anti-autoimmune diseases and organ transplantation survival (Zhang et al., 2016). Studies have shown that PD-L1 is a protein biomarker over expression in many lung tumors (Dholaria et al., 2016; D'Incecco et al., 2015). It plays an important role in lung tumors diagnosis, screening, evaluation of therapeutic efficacy and prognosis. Currently, the technique for determining the level of PD-L1 are mainly including western blot, enzyme-linked immunosorbent assay, immunobeads-based flow cytometry, SELDI-MS or MALDI-MS (Grasso et al., 2015; Muhammad et al.,2014; Lewis et al., 2018) and biosensors techniques (including immunofluorescence, colorimetric, surfaceenhanced Raman scattering, electrochemical, nuclear magnetic resonance and surface plasmon resonance biosensor) (He et al., 2014; Zhang et al.,2016; Xia et al., 2017; Zong et al., 2016; Doldán et al., 2016; Zhou et al., 2016). Among these techniques, biosensors have been introduced as alternative technologies to overcome other methods disadvantages; such as the kit is expensive, time-consuming and has the complex operation steps. Biosensor is rapid, highly sensitive and throughput and real-time optical detection of PD-L1. According to the relevant data, it has been proved that many biosensors have great application prospects in cancer diagnosis and testing; in particular, fluorescence-based methods biosensor have attracted special attention because of their low sample volume, easy operation, sensitivity and high specificity.

In recent years, there are some reports of immunofluorescence biosensor based on aptamers and deoxyribonuclease I (DNase I); aptamer is a single-stranded oligonucleotides, generated by a SELEX process, and can specifically bind to small molecules, cells and proteins (Jin et al., 2017; Jun et al.,2018). Aptamers are high stability, low cost and easy chemical synthesis; thus, it has been widely used for biomarker discovery and biosensing fields (Yuan et al., 2017; Lan et al.,2013). DNase I is an enzyme-aided signal amplification, which can cleave phosphodiester bonds to product many polynucleotides with 5-phosphate and 3'-OH groups but cannot digest RNA sequences (Lagardère et al., 2017; $\mathrm{Li}$ et al.,2017). Thus, it has been developed to achieve the sensitive detection of biomolecules recently. In addition, the application of nanomaterials in fluorescent biosensors has become an advancing field, such as organic dyes, quantum dots, noble metal nanoclusters, carbon dots and graphene oxide (GO) (Zhang et al., 2012; Xu et al., 2015; Yin et al., 2015; Rizwan et al., 2018). GO is a one-atom-thick two-dimensional carbon nanomaterial with a honeycomb structure (Liu et al., 2016), which has attracted remarkable attention due its unique quenching fluorescence ability as well as adsorption capacity for oligonucleotides. However, in some studies, it was found that the fluorescent biosensor based on GO also has disadvantages, such as, poor photostability and low solubility in water. Thus, it is necessary to search for better fluorophors in biosensor.

In this work, we take the advantages of reduced graphene oxide (rGO) and magnetic silicon microspheres (MNPs) to propose a sensitivity and specificity assay for the quantitative detection of PD-L1 in lung tumors plasma. MNPs have good biocompatibility, easy separation, and ample functional groups for modification (Martín et al., 2015; Altintas, , 2017), thus its can connect capture probes, separate targets, and rGO from substances (Zhang et al., 2014), rGO, the strong absorbing capability of single-stranded oligonucleotides and weak interaction with double-stranded DNA-RNA hybrids (Joon Soo et al., 2014), more importantly, nano-materials will have inner filter effect in solution, the magnetic nanoparticles modified GO can be separated from solution by magnetic field to avoid inner filter effect, thus reduce its interference on the sample fluorescence, because the presence of particles in the solution will reduce the fluorescence intensity (Altintas, , 2017). Based on the above advantages, in the absence of PD-L1, PD-L1 aptamer (Apt) could be absorbed by rGO-MNPs, so that the effective fluorescence quenching. Correspondingly, in the presence of PD-L1, because of double-stranded DNA-RNA hybrids, the Apt/PD-L1 complex was away from rGO-MNPS and resulting in the recovery of quenched fluorescence. When DNase I is added, it can cleave phosphodiester as well as enzyme-aided signal amplification, leading to the release of PD-L1 and FAM fluorophores, so that the accumulation of free FAM fluorophores and the quantities of PD-L1 can be achieved by fluorescence increment. With the detection range from $100 \mathrm{pg} \cdot \mathrm{mL}^{-1}$ to $100 \mathrm{ng} \cdot \mathrm{mL}^{-1}$, the detection limit was low at $10 \mathrm{pg} \cdot \mathrm{mL}^{-1}$. More importantly, the method has been successfully applied to the detection of PD-L1 in various spiked biological samples (including human urine, saliva, serum, lung cancer patients and normal controls plasma). We believe that this biosensor may be used as a clinical therapeutic and diagnosis tool for lung tumors.

\section{Experimental section}

\subsection{Reagents and materials}

The ExoQuick Plasma prep and Exosome precipitation kit (from System Biosciences, PM-EXOQ5TM-1). DNase I was obtained from solarbio (Beijing, China; www.solarbio.com). The rGOMNPS was purchased from Nanjing Xianfeng, as a reduced graphene oxide modified magnetic nanoparticles. The PD-L1, CA125, CD63, EPCAM and VEGF were purchased from Cusabio Biotech Co.Ltd. (www.cusabio.cn/). The PD-L1 aptamer (5'-ACG GGC CAC ATC AAC TCA TTG ATA GAC AAT GCG TCC ACT GCC CGT-3'-FAM) was synthesized by Sangon Biological Engineering Technology Co., Ltd. (Shanghai, China; www.sangon. com), and purified using high performance liquid chromatography. All reagents were diluted to the required concentration with working buffer (10 mM Tris, $100 \mathrm{mM} \mathrm{NaNO}_{3} \mathrm{pH}$ 7.4) before usage. The ultrapure water obtained from a millipore water purification system $(18.2 \mathrm{M} \Omega \cdot \mathrm{cm}$ resistivity, Milli-Q Direct 8).

\subsection{Human samples}

In this study, human plasma samples were collected from nonsmall cell lung cancer patients prior to treatment and healthy donors. Healthy human serum, urine and saliva were obtained from Affiliated Dongfeng Hospital, Hubei University of Medicine, and approved by Hospital's Ethics Committee. We selected the exosome surface proteins PD-L1 as the biomarkers in this study. The steps of exosome separation are as follows: exosomes were extracted and purified following the Exo-Quick ${ }^{\mathrm{TC}}$ Exosome Isolation Reagent manufacturer's protocol with minor revision. In brief, $400 \mathrm{uL}$ human plasma samples was taken, the PEG in the exosome isolation kit was added into the plasma sample after centrifugation in the proportion of $1: 4$, mixture was incubated at $4^{\circ} \mathrm{C}$ for $30 \mathrm{~min}$ and $1,500 \mathrm{~g}$ centrifuge for $30 \mathrm{~min}$ at $4^{\circ} \mathrm{C}$. The supernatant was removed, and the exosome pellet was resuspended in $400 \mu \mathrm{L}$ PBS. The samples are divided into four 
parts and stored at $-80^{\circ} \mathrm{C}$; after the experiment, $100 \mu \mathrm{L}$ exosome was taken as the sample to be tested.

\subsection{Optimization of sensing conditions}

The fluorescence of the mixture was carried out on a Hitachi F4600 spectrophotometer (Hitachi Co. Ltd, Japan) equipped with a xenon lamp excitation source at room temperature. The excitation was set at $495 \mathrm{~nm}$, and the emission spectra were collected from $510 \mathrm{~nm}$ to $600 \mathrm{~nm}$. In this part, the $40 \mathrm{nM}$ FAM labeled PD-L1 Apt and a desired concentration of PD-L1 were first mixed and kept at $37^{\circ} \mathrm{C}$ for $15 \mathrm{~min}$, and then $60 \mu \mathrm{g} \cdot \mathrm{mL}^{-1}$ of rGO-MNPS was added. About after $5 \mathrm{~min}$, the reaction solution was added with $6 \mathrm{U} \cdot \mathrm{mL}^{-1} \mathrm{DNase} \mathrm{I}$ and incubated at $37^{\circ} \mathrm{C}$ for $30 \mathrm{~min}$. Then the solution was diluted to $1 \mathrm{~mL}$. Finally, the fluorescence intensity at $517.6 \mathrm{~nm}$ was used to choose the optimal experimental conditions and quantitative determination of PD-L1. In control experiments, measurement of fluorescence intensity at $517.6 \mathrm{~nm}$ in the absence of PD-L1. Unless otherwise noted, each fluorescence measurement was repeated three times, and the standard deviation was plotted as the error bar.

\section{Results and discussion}

3.1 Characterization of reduced graphene oxide-MNPS The morphology of the obtained samples was characterized by transmission electron microscopy as shown in Figure 1. The high resolution transmission electron microscopy images were acquired by a Tecnai G2 F30, and transmission electron microscope (FEI, USA) operating at an acceleration voltage of $300 \mathrm{kV}$. These results clearly indicated the successful synthesis of rGO-MNPS.

\subsection{Principle for detection of PD-L1}

As shown in Figure 2, based on the strong absorb capability of rGO with single-stranded oligonucleotides via $\pi-\pi$ stacking interaction, but weak interaction with double-stranded DNARNA hybrids (Huang and Liu, 2013). Thus, in the absence of PD-L1, the rGO-MNPS enabled strong adsorption of Apt to the surface of rGO-MNPS, leading to the effective fluorescence quenching. Correspondingly, in the presence of PD-L1, because of double-stranded DNA-RNA hybrids, the Apt/PD-L1 complex was away from rGO-MNPS and resulting in the recovery of quenched fluorescence. Meanwhile, when the DNase I is added, based on the DNase I enzyme-aided signal amplification and leading to the release of PD-L1 and FAM fluorophores. The released PD-L1 is recycled repeatedly, resulting in the accumulation of free FAM fluorophores. The quantities of PDL1 can be achieved by fluorescence increment.

Figure 1 TEM images of rGO-MNPS

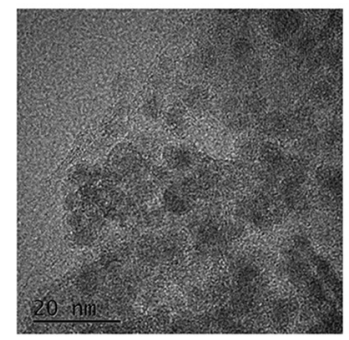

(a)

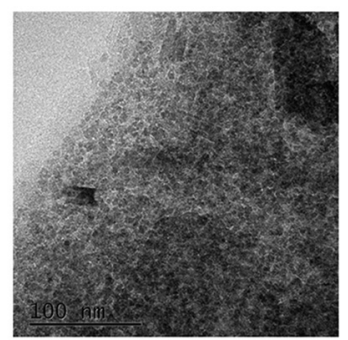

(b)
Figure 2 Schematic illustration of the fluorescent biosensor assay detection of PD-L1 by using rGO-MNPS and DNase I-mediated target cyclic amplification

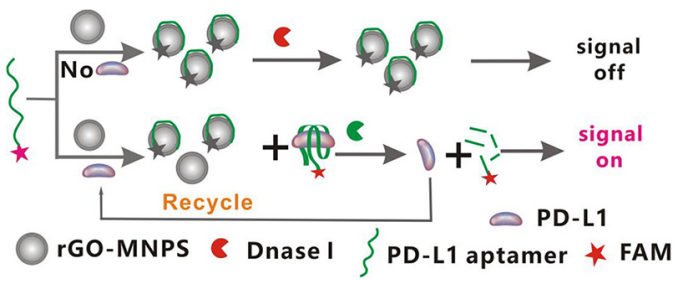

\subsection{Feasibility for strategy}

To investigate the feasibility of the biosensor for PD-L1 detection, Figure 3 shows the fluorescence emission spectra of the Apt under different conditions. Which acted as the control experiment (curve a), displayed a strong fluorescence emission when $30 \mathrm{nM}$ Apt added alone. The introduction of rGOMNPS quenched the fluorescence emission (curve d), resulting in a fluorescence intensity decreased remarkably, shows the rGO-MNPS enabled strong adsorption of Apt and effective fluorescence quenching. Then DNase I was added to a solution of Apt and rGO-MNPS, the fluorescence intensity not obviously increase (curve c), indicating that Apt cannot specifically bind to PD-L1, lead to the FAM fluorophores are produced of the experiment. However, upon adding PD-L1, a significant enhancement of the fluorescence intensity was observed (curve b), as the result of the stronger binding affinity between PD-L1 and their corresponding aptamers induced unwinding of PD-L1 Apt from the rGO-MNPS surface. More importantly, the DNase I-aided target recycling results in the significant fluorescence amplification. Taking these results together, the feasibility of the proposed aptasensor for PD-L1 detection by our sensing method was achieved.

\subsection{Optimization of experimental conditions}

The optimal performance of the fluorescent sensing protocol strongly affected the main experimental conditions, such as the

Figure 3 Feasibility analysis of the biosensor

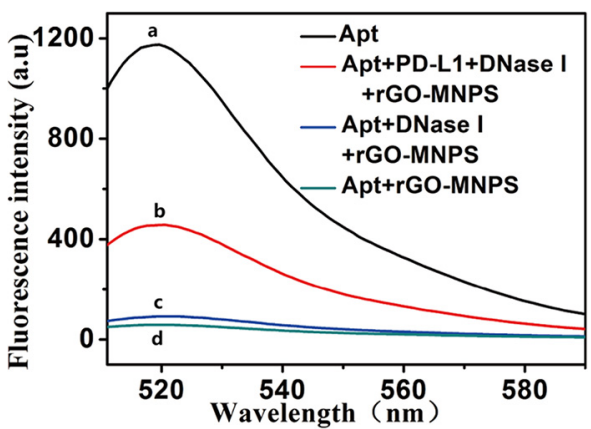

Notes: (a) Apt; (b) Apt + PD-L1 + DNase I + rGO-MNPS; (c) Apt + DNase I + rGOMNPS; (d) Apt + rGO-MNPS. The concentrations of Apt, PD-L1, DNase I and rGO-MNPS were $40 \mathrm{nM}, 10 \mathrm{ng} \cdot \mathrm{mL}^{-1}, 6$ $\mathrm{U} \cdot \mathrm{mL}^{-1}$ and $60 \mu \mathrm{g} \cdot \mathrm{mL}^{-1}$, respectively 
concentration of rGO-MNPS and DNase I, the enzyme digestion reaction time and the reaction temperature. The $\mathrm{F}_{1}$ and $F_{0}$ represent the fluorescence intensity of the solution in the presence and absence of PD-L1, respectively. As shown in Figure 4(a), Maximum $F_{1} / F_{0}$ value was observed when the concentration of rGO-MNPS was $60 \mu \mathrm{g} \cdot \mathrm{mL}^{-1}$, when increasing the concentration of rGO-MNPS; however, $\mathrm{F}_{1} / \mathrm{F}_{0}$ value decreases obviously along with the increasing of rGOMNPS concentration. Thus, the concentration of rGO-MNPS of $60 \mu \mathrm{g} \cdot \mathrm{mL}^{-1}$ was confirmed as the optimized concentration. According to Figure $4(\mathrm{~b})$, maximum $\mathrm{F}_{1} / \mathrm{F}_{0}$ values are observed when the concentration of DNase I is $6 \mathrm{U} \cdot \mathrm{mL}^{-1}$ and then decreased gradually because of an accelerated increase in background fluorescent signal; therefore, $6 \mathrm{U} \cdot \mathrm{mL}^{-1}$ is selected as the optimum DNase I concentration.

In addition, Figure 4 (c) shows the effect of enzyme digestion reaction time on the fluorescence intensity at the emission wavelength of $517.6 \mathrm{~nm}$. The fluorescence intensity and the value of $F_{1} / F_{0}$ is related to the enzyme digestion reaction time; the value of $F_{1} / F_{0}$ increased gradually along with the increasing enzyme digestion reaction time in the range from 5 to $30 \mathrm{~min}$, and the maximum $\mathrm{F}_{1} / \mathrm{F}_{0}$ values are observed when the enzyme digestion time is $30 \mathrm{~min}$. Thus, $30 \mathrm{~min}$ was selected as the optimal hybridization time. According to Figure $4(d)$, the $F_{1} / F_{0}$ value was affected at different levels with the rise of the hybridization temperature; the $\mathrm{F}_{1} / \mathrm{F}_{0}$ value reached a maximum when enzyme digestion temperature was $37^{\circ} \mathrm{C}$ and then decreased gradually; thus, $37^{\circ} \mathrm{C}$ of enzyme digestion reaction temperature was selected for the rest of the experiments.

Figure 4 (a) and (b) shows the effect of rGO-MNPS and DNase I concentration on the fluorescence response of this method, respectively; (c) and (d) shows the effect of Enzyme digestion reaction time and reaction temperature on the fluorescence intensity at the emission wavelength of $517.6 \mathrm{~nm}$, respectively

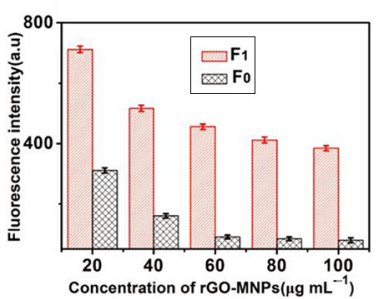

(a)

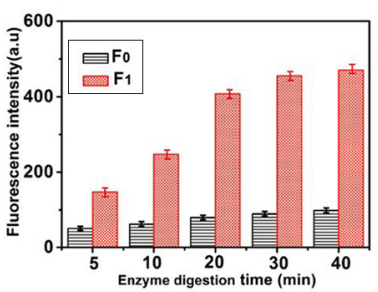

(c)

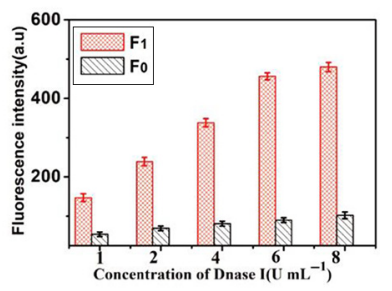

(b)

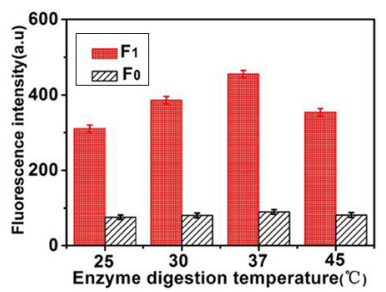

(d)
Notes: $F_{0}$ : in the absence of PD-L1; $F_{1}$ : in the present of PD-L1, and the concentrations of Apt, PD-L1, DNase I and rGO-MNPS were $40 \mathrm{nM}, 10 \mathrm{ng} \cdot \mathrm{mL}^{-1}, 6 \mathrm{U} \cdot \mathrm{mL}^{-1}$ and $60 \mu \mathrm{g} \cdot \mathrm{mL}^{-1}$, respectively, Error bars: SD, $n=3$

\subsection{Sensitivity and specificity}

To investigate the sensitivity and specificity of the proposed sensing strategy, various concentrations of PD-L1 were measured under the optimized experimental conditions. As shown in Figure 5(a), increasing fluorescence intensity with increasing PD-L1 concentration from 0 to $200 \mathrm{ng} \cdot \mathrm{mL}^{-1}$. It illustrates a highly concentration dependence of the sensor for the detection of PD-L1. Notably, a good linear correlation between the fluorescence intensity and the concentration of PD-L1 in the range from $100 \mathrm{pg} \cdot \mathrm{mL}^{-1}$ to $100 \mathrm{ng} \cdot \mathrm{mL}^{-1}$ [shown in Figure 5(b)]. The calibration plot of the linear equation is given as $\mathrm{y}=137.91 \cdot \lg \mathrm{c}-112.89\left(R^{2}=0.9935\right)$, where $\lg \mathrm{c}$ is the logarithm of PD-L1 concentration and $\mathrm{Y}$ is the fluorescence intensity. Furthermore, the detection limit is low to $10 \mathrm{pg} \cdot \mathrm{mL}^{-1}$. In addition, the selectivity of this detection strategy was assessed by measuring and recording the fluorescence response of other different targets such as CA125, CD63, VEGF and EPCAM; Figure 6 displays the high fluorescence signal in the presence of the PD-L1 $\left(10 \mathrm{ng} \cdot \mathrm{mL}^{-1}\right)$ with in the presence of other control proteins $\left(50 \mathrm{ng} \cdot \mathrm{mL}^{-1}\right)$. This comparison clearly indicates the high selectivity of our sensing method.

\subsection{Analysis of PD-L1 in real samples}

To further assess the effectiveness of the sensor in biological samples, the detection of PD-L1 in real samples was carried out. First, the detection of PD-L1 was spiked with $10 \%$ diluted with buffer solution to human urine, saliva and serum, and the final concentration of $10 \mathrm{ng} \cdot \mathrm{mL}^{-1}$ was performed. Herein, a significant increase of fluorescence in the presence of $10 \mathrm{ng} \cdot \mathrm{mL}^{-1} \mathrm{PD}-\mathrm{L} 1$ in various biological samples, compared with no spiking biological samples[shown in Figure 7(a)]. In addition, as shown in Figure 7(b), higher PD-L1 levels were observed in serum samples from lung cancer patients than healthy controls, suggesting that maybe PD-L1 is an important biomarker for lung cancer diagnosis. In summary, these results demonstrate that this sensor has considerable application potential to analyze biomarkers in real biological samples, and

Figure 5 (a) Fluorescence emission spectra of the biosensor in the presence of PD-L1 with different concentrations: from bottom to top: 0 , $10,100,500,1,000,5,000,10,000,50,000,100,000$ and $200,000 \mathrm{pg} \cdot \mathrm{mL}^{-1}$, respectively. (b) The relationship curve of the fluorescence intensity as a function of PD-L1 concentration

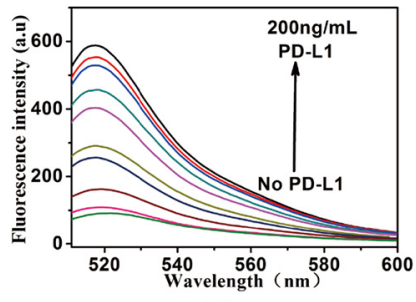

(a)

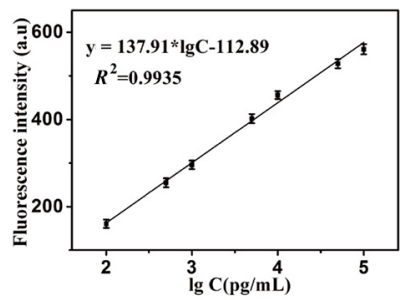

(b)
Notes: While shows the relationship between fluorescence intensity and PD-L1 concentration, both experimental conditions: Apt, PD-L1, DNase I and rGO-MNPS were $40 \mathrm{nM}$, $10 \mathrm{ng} \cdot \mathrm{mL}^{-1}, 6 \mathrm{U} \cdot \mathrm{mL}^{-1}$ and $60 \mu \mathrm{g} \cdot \mathrm{mL}^{-1}$, respectively; and the emission wavelength of $517.6 \mathrm{~nm}$. Error bars: SD, $n=3$ 
Figure 6 Fluorescence intensity (at the emission wavelength of $517.6 \mathrm{~nm})$ of the sensor in the presence of PD-L1 $\left(10 \mathrm{ng} \cdot \mathrm{mL}^{-1}\right)$, CA125 $\left(50 \mathrm{ng} \cdot \mathrm{mL}^{-1}\right), \quad$ CD63 $\left(50 \mathrm{ng} \cdot \mathrm{mL}^{-1}\right), \quad$ VEGF $\left(50 \mathrm{ng} \cdot \mathrm{mL}^{-1}\right), \quad$ EPCAM (50 $\left.\mathrm{ng} \cdot \mathrm{mL}^{-1}\right)$ and black, respectively

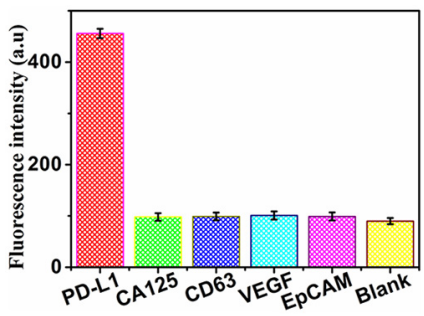

Note: Error bars: SD, $n=3$

Figure 7 Fluorescence intensity of the sensor for detection of healthy controls and LC patients, and detection of the PD-L1 in buffer and blank biological samples (human urine, saliva and serum, respectively)

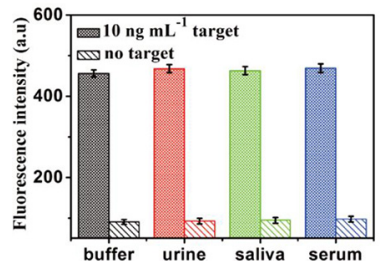

(a)

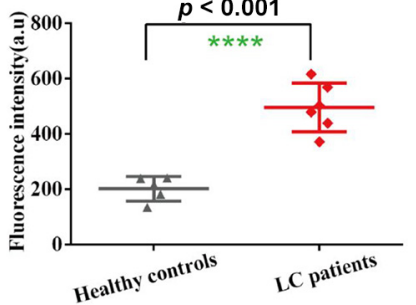

(b)
Note: Error bars: SD, $n=3$

it may serve as an assay that assists in cancer diagnosis, screening and prognosis.

\section{Conclusions}

Based on DNase I and rGO-MNPS, a highly sensitive and selective fluorescent probe for the detection of PD-L1 was developed. In this design, as the concentration of rGOMNPS and DNase I, the enzyme digestion reaction time and reaction temperature were optimized, the sensitive and specific detection of PD-L1 was achieved; this sensor can detect PD-L1 with a linear range from $100 \mathrm{pg} \cdot \mathrm{mL}^{-1}$ to $100 \mathrm{ng} \cdot \mathrm{mL}^{-1}$ and a detection limit of $10 \mathrm{pg} \cdot \mathrm{mL}^{-1}$. Then, the fluorescence signals are also obtained when the proposed strategy is used in biological samples; moreover, the use of DNase I and rGO-MNPs has developed an interference-free fluorescence measurement biosensor; we believed that this fluorescent method provides promising application in biomarker analysis.

\section{References}

Aberle, D.R., Abtin, F. and Brown, K. (2013), "Computed tomography screening for lung cancer: has it finally arrived? Implications of the national lung screening trial", fournal of Clinical Oncology, Vol. 31 No. 8, pp. 1002-1008.
Altintas, Z. (2017), “Applications of magnetic nanomaterials in biosensors and diagnostics", Biosensors and Nanotechnology: Applications in Health Care Diagnostics, Vol. 73, pp. 277-296.

Chudgar, N.P., Bucciarelli, P.R., Jeffries, E.M., Rizk, N.P., Park, B.J., Adusumilli, P.S. and Jones, D.R. (2015), "Results of the national lung cancer screening trial: where are we now?", Thoracic Surgery Clinics, Vol. 25 No. 2, pp. 145-153.

Dholaria, B., Hammond, W., Shreders, A. and Lou, Y. (2016), "Emerging therapeutic agents for lung cancer", fournal of Hematology \& Oncology, Vol. 9 No. 1, p. 138.

D'Incecco, A., Andreozzi, M., Ludovini, V., Rossi, E., Capodanno, A., Landi, L., Tibaldi, C., Minuti, G., Salvini, J. and Coppi, E. (2015), "PD-1 and PD-L1 expression in molecularly selected non-small-cell lung cancer patients", British Fournal of Cancer, Vol. 112 No. 1, pp. 95-102.

Doldán, X., Fagúndez, P., Cayota, A., Laíz, J. and Tosar, J.P. (2016), "Electrochemical sandwich immunosensor for determination of exosomes based on surface markermediated signal amplification", Analytical Chemistry, Vol. 88 No. 21, p. 10466.

Grasso, L., Wyss, R., Weidenauer, L., Thampi, A., Demurtas, D., Prudent, M., Lion, N. and Vogel, H. (2015), "Molecular screening of cancer-derived exosomes by surface plasmon resonance spectroscopy", Analytical and Bioanalytical Chemistry, Vol. 407 No. 18, pp. 5425-5432.

He, M., Crow, J., Roth, M., Zeng, Y. and Godwin, A.K. (2014), "Integrated immunoisolation and protein analysis of circulating exosomes using microfluidic technology", Lab on a Chip, Vol. 14 No. 19, pp. 3773-3780.

Huang, P.J. and Liu, J. (2013), "Separation of short single- and double-stranded DNA based on their adsorption kinetics difference on graphene oxide", Nanomaterials, Vol. 3 No. 2, pp. 221-228.

Jin, H., Gui, R., Sun, J. and Wang, Y. (2017), "Facilely selfassembled magnetic nanoparticles/aptamer/carbon dots nanocomposites for highly sensitive up-conversion fluorescence turn-on detection of tetrodotoxin", Talanta, Vol. 176, pp. 277-283.

Joon Soo, P., Nam-In, G. and Dong-Eun, K. (2014), "Mechanism of DNA adsorption and desorption on graphene oxide", Langmuir the ACS Fournal of Surfaces \& Colloids, Vol. 30, pp. 12587-12595.

Jun, Z., Fengying, R., Wenbo, Z., Bing, S., Fei, Y., Lun, W., Wanbao, H., Xueqin, H. Qinhua. and C, (2018), "Ultrasensitive fluorescent aptasensor for MUC1 detection based on deoxyribonuclease I-aided target recycling signal amplification", RSC Advances, Vol. 8, pp. 32009-32015.

Lagardère, L., Jolly, L.H., Lipparini, F., Aviat, F., Stamm, B., Jing, Z.F., Harger, M., Torabifard, H., Cisneros, G.A. and Schnieders, M.J. (2017), "Tinker-HP: a massively parallel molecular dynamics package for multiscale simulations of large complex systems with advanced point dipole polarizable force fields", Chemical Science, Vol. 9 No. 4, pp. 956-972.

Lan, H., Luo, Y., Zhi, W., Wu, Y. and Pei, Z. (2013), “A colorimetric aptamer biosensor based on gold nanoparticles for the ultrasensitive and specific detection of tetracycline in milk", Australian fournal of Chemistry, Vol. 66, pp. 485-490.

Lewis, J.M., Vyas, A.D., Qiu, Y., Messer, K.S. and Heller, M.J. (2018), "Integrated analysis of exosomal protein 
biomarkers on alternating current electrokinetic chips enables rapid detection of pancreatic cancer in patient blood", ACS Nano, Vol. 12 No. 4, pp. 3311-3320.

Li, D., Zhou, W., Yuan, R. and Xiang, Y. (2017), “A DNAfueled and catalytic molecule machine lights up trace underexpressed MicroRNAs in living cells", Analytical Chemistry (journal), Vol. 89 No. 18, pp. 9934-9940.

Lieping, C. and Flies, D.B. (2013), "Molecular mechanisms of T cell co-stimulation and co-inhibition", Nature Reviews Immunology, Vol. 13, pp. 227-242.

Liu, H., Tian, T., Ji, D., Ren, N., Ge, S., Yan, M. and Yu, J. (2016), "A graphene-enhanced imaging of microRNA with enzyme-free signal amplification of catalyzed hairpin assembly in living cells", Biosensors and Bioelectronics, Vol. 85, pp. 909-914.

Martín, M., González, O.A., Lorenzo-Luis, P., Hernández, C. A. González-Mora, J.L. and Salazar, P. (2015), "Quinonerich poly(dopamine) magnetic nanoparticles for biosensor applications", ChemPhysChem, Vol. 15 No. 17, pp. 3742-3752.

Muhammad, N., Giovanni, C., Hadi, V., Irina, N., Karin, E. M., Xiaoqin, W., Simona, P., Neelam, S., Ashraf, N.M. and Farah, F. (2014), "The emerging role of extracellular vesicles as biomarkers for urogenital cancers", Nature Reviews Urology, Vol. 11, pp. 688-701.

Oeffinger, K.C., Fontham, E.T.H., Ruth, E., Abbe, H., Michaelson, J.S., Ya-Chen Tina, S., Walter, L.C., Church, T.R., Flowers, C.R. and Lamonte, S.J. (2015), "Breast cancer screening for women at average risk: 2015 guideline update from the American cancer society", $\mathcal{F A M A}$, Vol. 314 No. 15, pp. 1599-1614.

Okazaki, T., Chikuma, S., Iwai, Y., Fagarasan, S. and Honjo, T. (2013), "A rheostat for immune responses: the unique properties of PD-1 and their advantages for clinical application", Nature Immunology, Vol. 14 No. 12, pp. 1212-1218.

Patz, E.F., Pinsky, P., Gatsonis, C., Sicks, J.D., Kramer, B.S., Tammem Gi, M.C., Chiles, C., Black, W.C. and Aberle, D.R. (2014), "Overdiagnosis in low-dose computed tomography screening for lung cancer", $\mathcal{F} A M A$ Internal Medicine, Vol. 174 No. 2, pp. 269-274.

Rizwan, M., Mohd-Naim, N.F. and Ahmed, M.U. (2018), "Trends and advances in electrochemiluminescence nanobiosensors", Sensors, Vol. 18 No. 2, pp. 1-28.

Xia, Y., Liu, M., Wang, L., Yan, A., He, W., Chen, M., Lan, J., $\mathrm{Xu}$, J., Guan, L. and Chen, J. (2017), "A visible and colorimetric aptasensor based on DNA-capped single-walled carbon nanotubes for detection of exosomes", Biosensors and Bioelectronics, Vol. 92, pp. 8-15.

Xu, R., Jiang, Y., Xia, L., Zhang, T., Xu, L., Zhang, S., Liu, D. and Song, H. (2015), "A sensitive photoelectrochemical biosensor for AFP detection based on $\mathrm{ZnO}$ inverse opal electrodes with signal amplification of CdS-QDs", Biosensors and Bioelectronics, Vol. 74, pp. 411-417.

Yin, H., Lei, J., Yan, C. and Ju, H. (2015), “Target-assistant $\mathrm{Zn} \mathrm{2+}$-dependent DNAzyme for signal-on electrochemiluminescent biosensing", Electrochimica Acta, Vol. 155, pp. 341-347.

Yuan, B., Jiang, X., Chen, Y., Guo, Q., Wang, K., Meng, X., Huang, Z. and Wen, X. (2017), "Metastatic cancer cell and tissue-specific fluorescence imaging using a new DNA aptamer developed by Cell-SELEX", Talanta, Vol. 170, p. 56.

Zhang, P., He, M. and Zeng, Y. (2016), "Ultrasensitive microfluidic analysis of circulating exosomes using a nanostructured graphene oxide/polydopamine coating", $L a b$ on a Chip, Vol. 16 No. 16, pp. 3033-3042.

Zhang, B., Chikuma, S., Hori, S., Fagarasan, S. and Honjo, T. (2016), "Nonoverlapping roles of PD-1 and FoxP3 in maintaining immune tolerance in a novel autoimmune pancreatitis mouse model", Proceedings of the National Academy of Sciences, Vol. 113 No. 30, pp. 8490-8495.

Zhang, M., Guo, S.M., Li, Y.R., Zuo, P. and Ye, B.C. (2012), "A label-free fluorescent molecular beacon based on DNAtemplated silver nanoclusters for detection of adenosine and adenosine deaminase", Chemical Communications, Vol. 48 No. 44, pp. 5488-5490.

Zhang, Y., Cheng, Y., Chen, N., Zhou, Y., Li, B., Gu, W., Shi, X. and Xian, Y. (2014), "Recyclable removal of bisphenol a from aqueous solution by reduced graphene oxide-magnetic nanoparticles: adsorption and desorption", fournal of Colloid and Interface Science, Vol. 421, pp. 85-92.

Zhou, Q., Rahimian, A., Son, K., Shin, D.S., Patel, T. and Revzin, A. (2016), "Development of an aptasensor for electrochemical detection of exosomes", Methods, Vol. 97, pp. 88-93.

Zong, S., Le, W., Chen, C., Ju, L., Dan, Z., Zhang, Y., Wang, Z. and Cui, Y.P. (2016), "Facile detection of tumor derived exosomes using magnetic nanobeads and SERS nanoprobes", Analytical Methods, Vol. 8 No. 25, pp. 5001-5008.

\section{Corresponding authors}

Qinhua Chen can be contacted at: cqh77@163.com and Fengying Ran can be contacted at: ranfengying13@163.com 